[Chem. Pharm. Bull.

[35( 5 )1785-1789(1987)]

\title{
The Constituents of Eucommia ulmoides OLIV. V. Isolation of Dihydroxydehydrodiconiferyl Alcohol Isomers and Phenolic Compounds
}

\author{
Takeshi Deyama, ${ }^{* a}$ Takako Ikawa, ${ }^{a}$ Shizuka Kitagawa, ${ }^{b}$ \\ and SANSEI NiSHIBE ${ }^{b}$ \\ Central Research Laboratories, Yomeishu Seizo, Co., Ltd., ${ }^{a}$ 2132-37 Naka-minowa, \\ Minowa-cho, Nagano 399-46, Japan and Faculty of Pharmaceutical Sciences, \\ Higashi Nippon Gakuen University," Ishikari-tobetsu, \\ Hokkaido 061-02, Japan
}

(Received September 22, 1986)

\begin{abstract}
Two new lignans, erythro-dihydroxydehydrodiconiferyl alcohol (1) and its threo isomer (2), were isolated from the bark of Eucommia ulmoides OLIV. (Eucommiaceae), together with six known lignans and three known phenolic compounds. Their structures were elucidated on the basis of spectroscopic analyses and chemical evidence.
\end{abstract}

Keywords-Eucommia ulmoides; lignan; dihydroxydehydrodiconiferyl alcohol; dihydrobenzofuran; phenolic compound; ${ }^{13} \mathrm{C}-\mathrm{NMR}$; HPLC

The bark of Eucommia ulmoides OLIV. (Eucommiaceae) is the origin of the crude drug, Eucommiae Cortex which has been used as a staminal tonic and hypotensor in China and Japan. In previous papers, we have reported the isolation of lignan glucosides ${ }^{1-4)}$ and iridoids. ${ }^{4)}$ We now wish to report the isolation of non-glucosidic phenolic compounds. Two new lignans, erythro-dihydroxydehydrodiconiferyl alcohol (1) and its threo isomer (2), were isolated from this crude drug, together with six known lignans, dihydrodehydrodiconiferyl alcohol (3), ${ }^{5)}(+)$-epipinoresinol (4), ${ }^{6)}(+)$-pinoresinol $(5),{ }^{1,7)}(+)$-medioresinol $(6),{ }^{1,7)}(+)$ syringaresinol $(7)^{1,7)}$ and $(+)$-1-hydroxypinoresinol $(\mathbf{8}),{ }^{2,6)}$ and three known phenolic compounds, erythro-and threo-guaiacylglycerol- $\beta$-coniferyl aldehyde ether $(\mathbf{9}),{ }^{8)}$ caffeic acid $(\mathbf{1 0})^{9)}$ and methyl chlorogenate (11), ${ }^{10)}$ and genipin (12). ${ }^{11)}$

The extraction and separation were carried out as described in the experimental section.

Compound 3 was isolated as a colorless syrup, $[\alpha]_{\mathrm{D}}^{25}+5.5^{\circ}(\mathrm{MeOH})$. Acetylation of 3 gave a triacetate (3a) as a colorless syrup, which gave peaks at $m / z 486\left(\mathbf{M}^{+}\right), 426$ $\left(\mathrm{M}^{+}-\mathrm{CH}_{3} \mathrm{COOH}\right)$ and 348 in the mass spectrum (MS). Compounds 3 and $\mathbf{3 a}$ were identified as dihydrodehydrodiconiferyl alcohol ${ }^{5)}$ and its triacetate, ${ }^{5)}$ respectively, by comparison with authentic samples.

Compound 9 was obtained as a colorless syrup, $[\alpha]_{\mathrm{D}}^{25}+20.0^{\circ}(\mathrm{MeOH})$, which gave peaks at $m / z 374\left(\mathrm{M}^{+}\right)$and $356\left(\mathrm{M}^{+}-\mathrm{H}_{2} \mathrm{O}\right)$ in the MS. The signals in the carbon-13 nuclear magnetic resonance $\left({ }^{13} \mathrm{C}-\mathrm{NMR}\right)$ spectrum of 9 were assigned as shown in Table $\mathrm{I}$, by comparison with those of $( \pm)$-erythro- and threo-guaiacylglycerol $(13),{ }^{3)}$ hedyotol $\mathrm{C}^{4,12)}$ and coniferyl alcohol. ${ }^{13)}$ Acetylation of 9 gave a triacetate (9a) as a colorless syrup. Compounds 9 and 9a were identified as erythro- and threo-guaiacylglycerol- $\beta$-coniferyl aldehyde ether ${ }^{8)}$ and its triacetate ${ }^{8)}$ by comparison with authentic samples.

Fraction A was obtained as a colorless syrup, $[\alpha]_{\mathrm{D}}^{26} 0^{\circ}(\mathrm{MeOH})$, which showed a spot at Rf 0.56 on thin layer chromatography (TLC) with $\mathrm{CHCl}_{3}-\mathrm{MeOH}$-water $(70: 30: 5)$ as a developing solvent. The MS of fraction A gave peaks at $m / z 392\left(\mathrm{M}^{+}\right), 374\left(\mathrm{M}^{+}-\mathrm{H}_{2} \mathrm{O}\right), 356$ 
$\left(\mathrm{M}^{+}-2 \times \mathrm{H}_{2} \mathrm{O}\right)$ and 344 . The proton nuclear magnetic resonance $\left({ }^{1} \mathrm{H}-\mathrm{NMR}\right)$ spectrum showed the signals due to two methoxyl groups ( $\delta 3.82$ and 3.88 ), and five aromatic protons ( $\delta 6.79$ and 6.93). The ${ }^{13} \mathrm{C}-\mathrm{NMR}$ chemical shifts were assigned as shown in Table I, by comparison with those of 13 and dehydrodiconiferyl aleohol ${ }^{5 b}(\mathbf{1 4})$. The shifts of the aromatic and $\alpha-, \beta$ - and $\gamma$-carbons of fraction $\mathrm{A}$ were in good agreement with those of $\mathbf{1 4}$. The signals of the $\mathrm{C}-\beta^{\prime}$ and $\mathrm{C}-\gamma$ atoms of fraction $\mathrm{A}$ appeared as doublets at $\delta 75.5$ and 76.1 , and at $\delta 64.3$ and 64.6, due to the erythro and threo isomers, respectively. From these findings, fraction A was concluded to be a mixture of erythro- and threo-dihydroxydehydrodiconiferyl alcohol. Fraction A was separated into two peaks (1 and 2) by high-performance liquid chromatography (HPLC). Peaks 1 and $\mathbf{2}$ were each isolated and purified by preparative HPLC, yielding 1 and 2. Compound 1 was obtained as a colorless syrup, $[\alpha]_{D}^{20} 0^{\circ}$ (water). The infrared (IR) spectrum showed the presence of hydroxyl groups $\left(3432 \mathrm{~cm}^{-1}\right)$ and aromatic rings (1610 and $1522 \mathrm{~cm}^{-1}$ ). The ultraviolet (UV) spectrum of 1 showed absorption maxima at 228, 283 and $288 \mathrm{~nm}$. The bathochromic shift of the absorption maxima in the presence of base was very similar to that of $\mathbf{3}$. Compound $\mathbf{2}$ was obtained as a colorless syrup, $[\alpha]_{\mathrm{D}}^{20^{\circ}} 0^{\circ}$ (water). The IR and UV spectra of $\mathbf{2}$ were very similar to those of $\mathbf{1}$. Acetylation of $\mathbf{1}$ and $\mathbf{2}$ gave pentaacetates, 1a and 2a, respectively. The MS of 1a and 2a were almost identical with each other, showing peaks at $m / z 602\left(\mathrm{M}^{+}\right)$and $542\left(\mathrm{M}^{+}-\mathrm{CH}_{3} \mathrm{COOH}\right)$. The ${ }^{1} \mathrm{H}-\mathrm{NMR}$ spectrum of 1a showed signals due to four alcoholic acetyl groups $(\delta 2.00,2.05$ and 2.13$)$, a phenolic acetyl group $(\delta 2.30)$, two aromatic methoxyl groups $(\delta 3.82$ and 3.91$)$, and five aromatic protons $(\delta 6.83$ and 6.99). That of $\mathbf{2 a}$ showed similar signals. It was reported that erythro-guaiacylglycerol tetraacetate and its threo isomer could be distinguished on the basis of the chemical shifts and the coupling constants of the benzylic protons. ${ }^{3,14)}$ The benzylic proton signals of 1a and $\mathbf{2 a}$ appeared at $\delta 5.93(\mathrm{~d}, J=5.72 \mathrm{~Hz})$ and $\delta 5.89(\mathrm{~d}, J=7.48 \mathrm{~Hz})$, which showed 1a and 2a to be the erythro and threo isomers, respectively. From the above results, compounds $\mathbf{1}$ and $\mathbf{2}$ were established to be erythro-dihydroxydehydrodiconiferyl alcohol and its threo isomer, respectively.<smiles>[R]OCC([R2])C([R])c1cc(OC)c2c(c1)C([R20])C(c1ccc([R20])c(OC)c1)O2</smiles>

$1: \mathrm{R}_{1}=\mathrm{H}, \mathrm{R}_{2}=\mathrm{OH}$ (erythro)

1a: $\mathrm{R}_{1}=\mathrm{Ac}, \mathrm{R}_{2}=\mathrm{OAc}$

$2: \mathrm{R}_{1}=\mathrm{H}, \mathrm{R}_{2}=\mathrm{OH}$ (threo)

$2 \mathrm{a}: \mathrm{R}_{1}=\mathrm{Ac}, \mathrm{R}_{2}=\mathrm{OAc}$

$3: \mathrm{R}_{1}=\mathrm{R}_{2}=\mathrm{H}$

$3 \mathbf{a}: \mathrm{R}_{1}=\mathrm{Ac}, \mathrm{R}_{2}=\mathrm{H}$

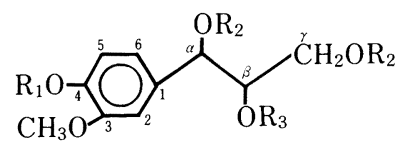

$$
\text { 9: } \mathrm{R}_{1}=\mathrm{R}_{2}=\mathrm{H}
$$

$13: \mathrm{R}_{1}=\mathrm{R}_{2}=\mathrm{R}_{3}=\mathrm{H}$
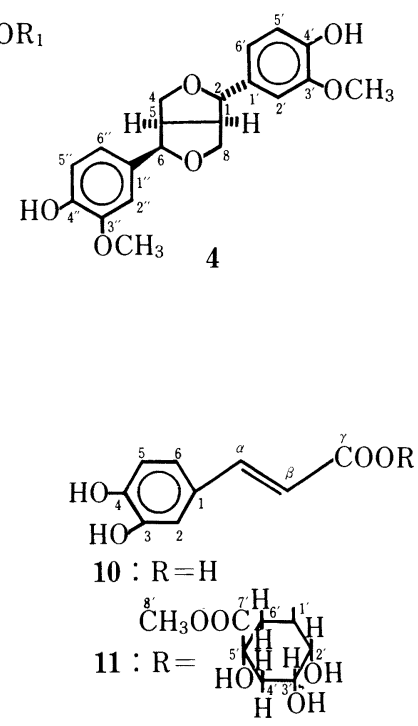
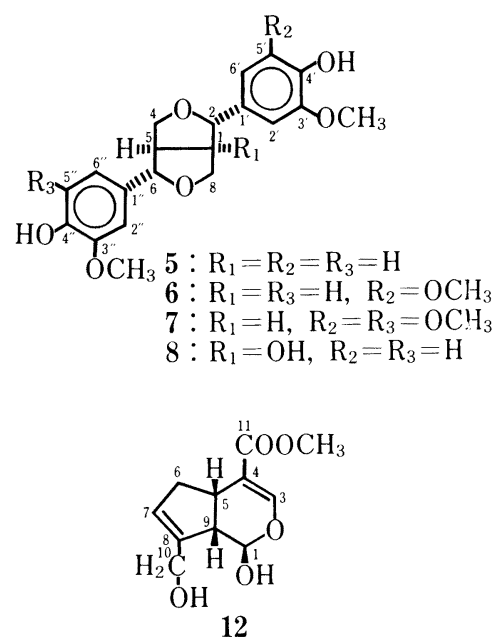

12 
TABLE I. ${ }^{13} \mathrm{C}-\mathrm{NMR}$ Chemical Shifts

\begin{tabular}{|c|c|c|c|c|c|}
\hline & $\begin{array}{l}\text { Fraction } \mathrm{A}^{a)} \\
\quad(\mathbf{1} \text { and } 2)\end{array}$ & $13^{a)}$ & $14^{b)}$ & $9^{c)}$ & $\begin{array}{c}\text { Guaiacylglycerol } \\
\text { moiety of } \\
\text { hedyotol } \mathrm{C}^{c)}\end{array}$ \\
\hline C-1 & 134.7 & 134.7 & 134.3 & $132.7,132.9$ & 133.2 \\
\hline$C-1^{\prime}$ & $136.8^{d)}$ & & 130.4 & 126.7 & \\
\hline $\mathrm{C}-2$ & 110.7 & $111.8,112.0$ & 110.7 & 111.2 & 111.2 \\
\hline C-2 & 112.9 & & 111.8 & 111.6 & \\
\hline C-3 & 148.9 & 148.8 & 148.6 & 146.9 & 147.0 \\
\hline$C-3^{\prime}$ & 145.2 & & 145.1 & $151.3,^{d)} 151.5^{d)}$ & \\
\hline C-4 & 147.6 & 147.1 & 147.5 & 145.5 & 145.4 \\
\hline C- $4^{\prime}$ & 149.1 & & 148.9 & $149.6^{d)}$ & \\
\hline C-5 & 116.1 & $115.8,115.9$ & 115.8 & 114.6 & 114.7 \\
\hline C- $5^{\prime}$ & $129.8^{d)}$ & & 132.0 & 123.2 & \\
\hline$C-6$ & 119.7 & $120.7,121.0$ & 119.5 & $119.0,119.6$ & 119.4 \\
\hline C- $6^{\prime}$ & 116.6 & & 116.2 & 114.6 & \\
\hline $\mathrm{OCH}_{3}$ & 56.4 & 56.5 & & 55.4 & 55.9 \\
\hline $\mathrm{OCH}_{3}$ & 56.8 & & & 55.7 & \\
\hline$\alpha$ & 89.1 & $76.6,{ }^{d)} 77.5$ & 88.3 & $71.1,71.6$ & 72.2 \\
\hline$\alpha^{\prime}$ & 77.4 & & 130.9 & 153.3 & \\
\hline$\beta$ & 55.2 & $76.1,^{d)} 75.5$ & 54.6 & $83.4,83.9$ & 86.1 \\
\hline$\beta^{\prime}$ & $75.5,76.1$ & & 128.0 & 126.3 & \\
\hline$\gamma$ & 64.8 & $64.3, \quad 64.5$ & 64.5 & 60.2 & 59.9 \\
\hline$\gamma^{\prime}$ & $64.3,64.6$ & & 63.3 & 193.8 & \\
\hline
\end{tabular}

a) In $\mathrm{MeOH}-d_{4} . \quad$ b) Data from ref. $5 b$, in $\mathrm{Me}_{2} \mathrm{CO}-d_{6}-\mathrm{D}_{2} \mathrm{O}(9: 1)$. c) In DMSO- $d_{6}$. d) The assignments may be interchanged in each column.

Compounds 4-8, 10, 11 and 12 were identified as $(+)$-epipinoresinol, ${ }^{6)}(+)$-pinoresinol, ${ }^{1,7)}(+)$-medioresinol, ${ }^{1,7)}(+)$-syringaresinol, ${ }^{1,7)}(+)$-1-hydroxypinoresinol, ${ }^{2,6)}$ caffeic acid, ${ }^{9)}$ methyl chlorogenate ${ }^{10)}$ and genipin ${ }^{11)}$ by comparison with authentic samples (see Experimental).

\section{Experimental}

All melting points are uncorrected. The following instruments were used; melting point, Mettler FP-61; optical rotation value, JASCO DIP-4; HPLC, Hitachi 638 with a UV detector; UV spectra, Hitachi 200-20, IR spectra, Hitachi 270-30; MS, Hitachi RMU-7L; ${ }^{1} \mathrm{H}-\mathrm{NMR}$ spectra, JEOL JNM-FX-90; ${ }^{13} \mathrm{C}-\mathrm{NMR}$ spectra, JEOL JNM-FX60 , with tetramethylsilane $(\delta=0)$ as an internal reference. The abbreviations used for NMR data are as follows: s, singlet; $\mathrm{d}$, doublet; $\mathrm{dt}$, doublet-triplet; $\mathrm{t}$, triplet; $\mathrm{m}$, multiplet.

Precoated TLC plates (Silica gel $60 \mathrm{~F}_{254}$, Merck) were used for TLC. The spots were detected by spraying the plates with $20 \% \mathrm{H}_{2} \mathrm{SO}_{4}$ and by heating. Silica gel (Wako gel C-300, Wako Pure Chemical Co.) and Diaion HP-20 (Nippon Rensui Co.) were used for column chromatography. TSK gel HW-40 (Toyo Soda Co.) was used for gel filtration.

Isolation - The air-dried bark of Eucommia ulmoides OLIV. (10 kg, commercial crude drug produced in China) was chopped and extracted with $50 \% \mathrm{MeOH}(201 \times 2)$ under reflux. The extract was filtered and the filtrate was evaporated under reduced pressure to a small volume, which was suspended in water. This suspension was extracted with EtOAc $(31 \times 2)$ and $n-\mathrm{BuOH}(31 \times 3)$, successively. The EtOAc extract was evaporated under reduced pressure to afford the residue, which showed spots at $R f 0.08$ [(+)-cyclo-olivil], 0.10 [(-)-olivil], $0.25(3), 0.33$ (9), 0.35 (12), 0.39 $(8), 0.53(5), 0.54(4), 0.55$ (6) and 0.56 (7) on TLC with $\mathrm{CHCl}_{3}-\mathrm{MeOH}(10: 1)$ as a developing solvent. This residue was subjected to silica gel chromatography with $\mathrm{CHCl}_{3}-\mathrm{MeOH}(40: 1,10: 1)$, and the eluate was separated into three fractions (frs. 1-3). Fraction 1 was applied to a silica gel column, and eluted successively with $\mathrm{CHCl}_{3}-\mathrm{MeOH}(20: 1)$, benzene-EtOAc $(5: 1,1: 1)$ and $n$-hexane-acetone $(1: 1)$. The eluates showing TLC spots at $R f 0.53(5), 0.54$ (4), 0.55 (6) and 0.56 (7) were each collected and purified by repeated silica gel chromatography and then gel filtration on TSK gel HW-40 with $\mathrm{MeOH}$, yielding $4(0.5 \mathrm{~g}), 5(3.5 \mathrm{~g}), 6(0.6 \mathrm{~g})$ and $7(0.4 \mathrm{~g})$. After repeated chromatography and gel filtration of fr. 2 and fr. 3 , under the same conditions, pure $8(1.0 \mathrm{~g}), \mathbf{9}(0.3 \mathrm{~g}), 12(2 \mathrm{~g})$ and $\mathbf{3}(0.1 \mathrm{~g})$ were isolated. The 
$n$ - $\mathrm{BuOH}$ extract was evaporated under reduced pressure to give the residue, which was subjected to silica gel chromatography with $\mathrm{CHCl}_{3}-\mathrm{MeOH}-$ water $(100: 10: 1,80: 20: 3)$. The eluates were monitored by TLC with $\mathrm{CHCl}_{3}-$ $\mathrm{MeOH}$-water $(70: 30: 5)$ as a developing solvent. The fractions showing a TLC spot at $R f 0.59$ (fraction A), 0.38 (10) and 0.11 (11) were each collected and purified by repeated silica gel chromatography and gel filtration on TSK gel $\mathrm{HW}-40$ with $\mathrm{MeOH}$, yielding fraction $\mathrm{A}(0.5 \mathrm{~g}), \mathbf{1 0}(0.1 \mathrm{~g})$ and $11(0.1 \mathrm{~g})$, respectively. Fraction A was separated into two peaks at $t_{\mathrm{R}}(\mathrm{min}) 6.33(\mathbf{1})$ and 7.14 (2) by HPLC. Conditions: column. Hitachi gel $3056,4 \mathrm{~mm} \times 25 \mathrm{~cm}$; eluent, $\mathrm{CH}_{3} \mathrm{CN}$-water (15:85); flow rate, $1.0 \mathrm{ml} / \mathrm{min}$; detector, UV detector $(270 \mathrm{~nm})$. Peaks 1 and 2 were each collected by repeated HPLC under the above conditions, yielding $1(1.8 \mathrm{mg})$ and $2(2.0 \mathrm{mg})$.

Fraction A(1 and 2)-A colorless syrup, $[\alpha]_{\mathrm{D}}^{26} 0^{\circ}(c=0.11, \mathrm{MeOH}) . \mathrm{UV} i_{\max }^{\mathrm{MeOH}} \mathrm{nm}: 235,285 . \mathrm{IR} v_{\max }^{\mathrm{KBr}} \mathrm{cm}^{-1}: 3680$ $(\mathrm{OH}), 1608,1518$ (aromatic ring). MS $m / z: 392\left(\mathrm{M}^{+}\right), 374\left(\mathrm{M}^{+}-\mathrm{H}_{2} \mathrm{O}\right), 356\left(\mathrm{M}^{+}-2 \times \mathrm{H}_{2} \mathrm{O}\right), 344 .{ }^{1} \mathrm{H}-\mathrm{NMR}$ (in $\left.\mathrm{CD}_{3} \mathrm{OD}\right) \delta: 3.82,3.88\left(3 \mathrm{H}, 3 \mathrm{H}\right.$, each s, $\left.2 \times \mathrm{OCH}_{3}\right), 5.54\left(1 \mathrm{H}, \mathrm{d}, J=5.71 \mathrm{~Hz}, \mathrm{C}_{\alpha}-\mathrm{H}\right), 6.79,6.93(2 \mathrm{H}, 3 \mathrm{H}$, each s, arom. $\mathrm{H})$.

erythro-Dihydroxydehydrodiconiferyl Alcohol (1)-A colorless syrup, $[\alpha]_{\mathrm{D}}^{20} 0 \quad(c=0.1$, water $)$. UV $i_{\max }^{\mathrm{E} \text { moH }} \mathrm{nm}$ : 228, 283, 288. UV $\lambda_{\max }^{\mathrm{EtOH}+\mathrm{NaOH}} \mathrm{nm}: 247,290 . \mathrm{IR} v_{\max }^{\mathrm{KBr}} \mathrm{cm}^{-1}: 3432(\mathrm{OH}), 1610,1522$ (aromatic ring). MS $m / z: 392\left(\mathrm{M}^{+}\right.$, 1), $374\left(\mathrm{M}^{+}-\mathrm{H}_{2} \mathrm{O}, 32\right), 356\left(\mathrm{M}^{+}-2 \times \mathrm{H}_{2} \mathrm{O}, 56\right), 344$ (100), $326(56), 314$ (90), 312 (60), $297(44), 285$ (78). ${ }^{1} \mathrm{H}-\mathrm{NMR}$ (in $\left.\mathrm{CD}_{3} \mathrm{OD}\right) \delta: 3.81,3.88\left(3 \mathrm{H}, 3 \mathrm{H}\right.$, each s, $\left.2 \times \mathrm{OCH}_{3}\right), 5.53\left(1 \mathrm{H}, \mathrm{d}, J=5.71 \mathrm{~Hz}, \mathrm{C}_{x}-\mathrm{H}\right), 6.78,6.93(2 \mathrm{H}, 3 \mathrm{H}$, each s, arom. $\mathrm{H}$ ).

threo-Dihydroxydehydrodiconiferyl Alcohol (2)-A colorless syrup, $[\alpha]_{\mathrm{D}}^{20} 0^{\circ}(c=0.15$, water $)$. UV $\lambda_{\mathrm{max}}^{\mathrm{EtOH}} \mathrm{nm}$ : 229, 282, 288. UV $i_{\max }^{\mathrm{EtOH}+\mathrm{NaOH}} \mathrm{nm}: 247.5,290 . \mathrm{IR} v_{\max }^{\mathrm{KBr}} \mathrm{cm}^{-1}: 3424(\mathrm{OH}), 1608,1520$ (aromatic ring). MS $\mathrm{m} / z: 392$ $\left(\mathrm{M}^{+}, 1\right), 374\left(\mathrm{M}^{+}-\mathrm{H}_{2} \mathrm{O}, 14\right), 356\left(\mathrm{M}^{+}-2 \times \mathrm{H}_{2} \mathrm{O}, 38\right), 345$ (60), 344 (56), 326 (44), 314 (92), 312 (100), $297(40), 285(58) .{ }^{1} \mathrm{H}-\mathrm{NMR}$ (in $\left.\mathrm{CD}_{3} \mathrm{OD}\right) \delta: 3.81,3.87\left(3 \mathrm{H}, 3 \mathrm{H}\right.$, each s, $\left.2 \times \mathrm{OCH}_{3}\right), 5.53\left(1 \mathrm{H}, \mathrm{d}, J=6.15 \mathrm{~Hz}, \mathrm{C}_{x}-\mathrm{H}\right)$, $6.75-7.00(5 \mathrm{H}, \mathrm{m}$, arom. $\mathrm{H})$. Compounds 1 and 2 were acetylated with acetic anhydride-pyridine, yielding the pentaacetates $1 \mathbf{a}$ and $\mathbf{2 a}$, respectively.

erythro-Dihydroxydehydrodiconiferyl Alcohol Pentaacetate (1a)-A colorless syrup. IR $v_{\max }^{\mathrm{KBr}} \mathrm{cm}^{-1}: 1764,1224$ $\left(\mathrm{OCOCH}_{3}\right), 1610,1506$ (aromatic ring). ${ }^{1} \mathrm{H}-\mathrm{NMR}\left(\right.$ in $\left.\mathrm{CDCl}_{3}\right) \delta: 2.00,2.05,2.13(3 \mathrm{H}, 6 \mathrm{H}, 3 \mathrm{H}$, each s, $4 \times$ alcoholic $\left.\mathrm{OCOCH}_{3}\right), 2.30\left(3 \mathrm{H}, \mathrm{s}\right.$, a phenolic $\left.\mathrm{OCOCH}_{3}\right), 3.82,3.91\left(3 \mathrm{H}, 3 \mathrm{H}\right.$, each s, $\left.2 \times \mathrm{OCH}_{3}\right), 5.53\left(1 \mathrm{H}, \mathrm{d}, J=7.03 \mathrm{~Hz}, \mathrm{C}_{x}-\mathrm{H}\right)$, $5.93\left(1 \mathrm{H}, \mathrm{d}, J=5.72 \mathrm{~Hz}, \mathrm{C}_{x^{\prime}} \mathrm{H}\right.$, erythro $), 6.83,6.99\left(2 \mathrm{H}, 3 \mathrm{H}\right.$, each s, arom. H). MS $m / z: 602\left(\mathrm{M}^{+}, 64\right), 542$ $\left(\mathrm{M}^{+}-\mathrm{CH}_{3} \mathrm{COOH}, 60\right), 500\left(\mathrm{M}^{+}-\mathrm{CH}_{3} \mathrm{COOH}-\mathrm{CH}_{2} \mathrm{CO}, 100\right), 482\left(\mathrm{M}^{+}-2 \times \mathrm{CH}_{2} \mathrm{COOH}, 17\right), 440$ (20).

threo-Dihydroxydehydrodiconiferyl Alcohol Pentaacetate (2a)-A colorless syrup. IR $v_{\max }^{\mathrm{KBr}} \mathrm{cm}^{-1}: 1764,1228$ $\left(\mathrm{OCOCH}_{3}\right), 1610,1506$ (aromatic ring). ${ }^{1} \mathrm{H}-\mathrm{NMR}$ (in $\left.\mathrm{CDCl}_{3}\right) \delta: 2.03,2.07,2.08(12 \mathrm{H}$, each s, $4 \times$ alcoholic $\left.\mathrm{OCOCH}_{3}\right), 2.31\left(3 \mathrm{H}, \mathrm{s}\right.$, a phenolic $\left.\mathrm{OCOCH}_{3}\right), 3.82,3.91\left(3 \mathrm{H}, 3 \mathrm{H}\right.$, each s, $\left.2 \times \mathrm{OCH}_{3}\right), 5.54\left(1 \mathrm{H}, \mathrm{d}, J=7.03 \mathrm{~Hz}, \mathrm{C}_{x}-\mathrm{H}\right)$, $5.89\left(1 \mathrm{H}, \mathrm{d}, J=7.48 \mathrm{~Hz}, \mathrm{C}_{\alpha^{\prime}}-\mathrm{H}\right.$, threo $), 6.83,6.99\left(2 \mathrm{H}, 3 \mathrm{H}\right.$, each s, arom. H). MS $m / z: 602\left(\mathrm{M}^{+}, 52\right), 542$ $\left(\mathrm{M}^{+}-\mathrm{CH}_{3} \mathrm{COOH}, 46\right), 500\left(\mathrm{M}^{+}-\mathrm{CH}_{3} \mathrm{COOH}-\mathrm{CH}_{2} \mathrm{CO}, 100\right), 440$ (12).

Dihydrodehydrodiconiferyl Alcohol (3)-A colorless syrup, $[\alpha]_{\mathrm{D}}^{25}+5.5^{c}(c=0.18, \mathrm{MeOH})$. UV $i_{\max }^{\mathrm{ErOH}} \mathrm{nm}: 228$, 283, 288. UV $i_{\max }^{\mathrm{EtOH}+\mathrm{NaOH}} \mathrm{nm}: 248,293 . \mathrm{IR} v_{\max }^{\mathrm{KBr}} \mathrm{cm}^{-1}: 3408(\mathrm{OH}), 1608,1520,1502$ (aromatic ring). ${ }^{1} \mathrm{H}-\mathrm{NMR}$ (in $\left.\mathrm{CDCl}_{3}\right) \delta: 1.60-2.10\left(3 \mathrm{H}, \mathrm{m}, \mathrm{C}_{\beta}-\mathrm{H}, \mathrm{C}_{\beta^{\prime}}-\mathrm{H}\right), 2.50-2.75\left(2 \mathrm{H}, \mathrm{m}, \mathrm{C}_{x^{\prime}}-\mathrm{H}\right), 3.40-4.00\left(2 \mathrm{H}, \mathrm{m}, \mathrm{C}_{;}-\mathrm{H}\right), 3.66(2 \mathrm{H}, \mathrm{t}$, $\left.J=6.6 \mathrm{~Hz}, \mathrm{C}_{-}-\mathrm{H}\right), 3.83,3.86\left(3 \mathrm{H}, 3 \mathrm{H}\right.$, each s, $\left.2 \times \mathrm{OCH}_{3}\right), 5.51\left(1 \mathrm{H}, \mathrm{d}, J=7.47 \mathrm{~Hz}, \mathrm{C}_{x}-\mathrm{H}\right), 6.65,6.70-6.80(2 \mathrm{H}, \mathrm{s}, 3 \mathrm{H}$, $\mathrm{m}$, arom. H). Acetylation of $\mathbf{3}$ with acetic anhydride-pyridine gave a triacetate (3a).

Dihydrodehydrodiconiferyl Alcohol Triacetate (3a)-A colorless syrup. IR $v_{\max }^{\mathrm{KBr}} \mathrm{cm}^{-1}: 1768,1740,1248$ $\left(\mathrm{OCOCH}_{3}\right), 1608,1506$ (aromatic ring). ${ }^{1} \mathrm{H}-\mathrm{NMR}$ (in $\left.\mathrm{CDCl}_{3}\right) \delta: 1.80-2.00\left(2 \mathrm{H}, \mathrm{m}_{,} \mathrm{C}_{\beta},-\mathrm{H}\right), 2.05,2.07(3 \mathrm{H}, 3 \mathrm{H}$, each $\mathrm{s}, 2 \times$ alcoholic $\left.\mathrm{OCOCH}_{3}\right), 2.31\left(3 \mathrm{H}, \mathrm{s}\right.$, a phenolic $\left.\mathrm{OCOCH}_{3}\right), 2.50-2.75\left(2 \mathrm{H}, \mathrm{m}, \mathrm{C}_{x^{\prime}}-\mathrm{H}\right), 3.82,3.90(3 \mathrm{H}, 3 \mathrm{H}$, each s, $\left.2 \times \mathrm{OCH}_{3}\right), 4.10\left(2 \mathrm{H}, \mathrm{t}, J=6.6 \mathrm{~Hz}, \mathrm{C}_{\cdots}-\mathrm{H}\right), 4.25-4.60\left(2 \mathrm{H}, \mathrm{m}, \mathrm{C}_{-}-\mathrm{H}\right), 5.52\left(1 \mathrm{H}, \mathrm{d}, J=6.6 \mathrm{~Hz}, \mathrm{C}_{x}-\mathrm{H}\right), 6.65,6.90-7.10$ $(2 \mathrm{H}, \mathrm{s}, 3 \mathrm{H}, \mathrm{m}$, arom. $\mathrm{H})$.

erythro- and threo-Guaiacylglycerol- $\beta$-coniferyl Aldehyde Ether (9)-A colorless syrup. $[\alpha]_{\mathrm{D}}^{25}+20.0 \quad(c=0.1$,

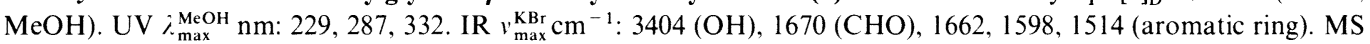
$m / z: 374\left(\mathrm{M}^{+}\right), 356\left(\mathrm{M}^{+}-\mathrm{H}_{2} \mathrm{O}\right), 326,204,178 .{ }^{1} \mathrm{H}-\mathrm{NMR}\left(\right.$ in $\left.\mathrm{CDCl}_{3}\right) \delta: 3.88,3.91\left(3 \mathrm{H}, 3 \mathrm{H}\right.$, each s, $\left.2 \times \mathrm{OCH}_{3}\right), 4.10-$ $4.40\left(1 \mathrm{H}, \mathrm{m}, \mathrm{C}_{\beta}-\mathrm{H}\right), 4.98\left(1 \mathrm{H}, \mathrm{d}, J=6.16 \mathrm{~Hz}, \mathrm{C}_{\alpha}-\mathrm{H}\right), 6.61,6.62\left(1 \mathrm{H} 2 \times \mathrm{dd}, J=7.48,15.39 \mathrm{~Hz}, \mathrm{C}_{\beta}-\mathrm{H}\right), 6.80-7.50(7 \mathrm{H}$, $\mathrm{m}, \mathrm{C}_{x^{\prime}}-\mathrm{H}$, arom. H), 9.66, $9.68(1 \mathrm{H}, 2 \times \mathrm{d}, J=7.48 \mathrm{~Hz}, \mathrm{CHO}) .{ }^{13} \mathrm{C}-\mathrm{NMR}$ : Table I. Acetylation of 9 gave a triacetate (9a).

erythro- and threo-Guaiacylglycerol- $\beta$-coniferyl Aldehyde Ether Triacetate (9a)-A colorless syrup. IR $v_{\max }^{\mathrm{KBr}} \mathrm{cm}^{-1}: 1746,1222\left(\mathrm{OCOCH}_{3}\right), 1678(\mathrm{CHO}), 1626,1600,1514$ (aromatic ring). ${ }^{1} \mathrm{H}-\mathrm{NMR}$ (in $\left.\mathrm{CDCl}_{3}\right) \delta: 1.99,2.03$, 2.06, $2.09\left(6 \mathrm{H}, 2 \times\right.$ alcoholic $\left.\mathrm{OCOCH}_{3}\right), 2.30\left(3 \mathrm{H}, \mathrm{s}\right.$, a phenolic $\left.\mathrm{OCOCH}_{3}\right), 3.83,3.88\left(3 \mathrm{H}, 3 \mathrm{H}\right.$, each s, $\left.2 \times \mathrm{OCH}_{3}\right)$, $3.95-4.45\left(2 \mathrm{H}, \mathrm{m}, \mathrm{C}_{\gamma}-\mathrm{H}\right), 4.60-4.90\left(1 \mathrm{H}, \mathrm{m}, \mathrm{C}_{\beta}-\mathrm{H}\right), 6.06,6.11\left(1 \mathrm{H}, 2 \times \mathrm{d}, J=5.0 \mathrm{~Hz}\right.$; erythro, $J=6.16 \mathrm{~Hz}$; threo $\mathrm{C}_{x}$ $\mathrm{H}), 6.61\left(1 \mathrm{H}, \mathrm{dd}, J=7.92,15.83 \mathrm{~Hz}, \mathrm{C}_{\beta^{\prime}}-\mathrm{H}\right), 6.90-7.20\left(6 \mathrm{H}, \mathrm{m}\right.$, arom. H), $7.40\left(1 \mathrm{H}, \mathrm{d}, J=15.83 \mathrm{~Hz}, \mathrm{C}_{x^{\prime}}-\mathrm{H}\right), 9.67(1 \mathrm{H}$, d, $J=7.92 \mathrm{~Hz}, \mathrm{CHO})$.

(+)-Epipinoresinol (4)—A white powder (from EtOH), mp $121^{\circ} \mathrm{C} .[\alpha]_{\mathrm{D}}^{25}+70.0 \quad(c=0.1, \mathrm{MeOH})$. UV $\lambda_{\max }^{\mathrm{MeOH}} \mathrm{nm}: 230,281$. IR $v_{\max }^{\mathrm{KBr}} \mathrm{cm}^{-1}: 3440(\mathrm{OH}), 1608,1516$ (aromatic ring). ${ }^{1} \mathrm{H}-\mathrm{NMR}$ (in dimethylsulfoxide- $d_{6}$ $\left.\left(\mathrm{DMSO}-d_{6}\right)\right) \delta: 3.76\left(6 \mathrm{H}, \mathrm{s}, 2 \times \mathrm{OCH}_{3}\right), 4.28\left(1 \mathrm{H}, \mathrm{d}, J=7 \mathrm{~Hz}, \mathrm{C}_{6}-\mathrm{H}\right), 4.74\left(1 \mathrm{H}, \mathrm{d}, J=5 \mathrm{~Hz}, \mathrm{C}_{2}-\mathrm{H}\right), 6.77-6.88(6 \mathrm{H}, \mathrm{m}$, arom. H) ${ }^{13} \mathrm{C}$-NMR (in DMSO- $\left.d_{6}\right) \delta: 49.5(5), 53.8(1), 55.8\left(\mathrm{OCH}_{3}\right), 68.4(4), 70.4(8), 81.4(6), 86.9(2), 110.1,110.5$ $\left(2^{\prime}, 2^{\prime \prime}\right), 115.2\left(5^{\prime}, 5^{\prime \prime}\right), 118.0,118.6\left(6^{\prime}, 6^{\prime \prime}\right), 129.7\left(1^{\prime \prime}\right), 132.5\left(1^{\prime}\right), 145.4,146.0\left(4^{\prime}, 4^{\prime \prime}\right), 147.3,147.5\left(3^{\prime}, 3^{\prime \prime}\right)$. MS $m / z$ : 
$358\left(\mathrm{M}^{+}, 88\right), 327$ (27), 205 (43), $196(26), 163$ (56), 151 (100), 137 (69), 131 (48), 124 (37)

(+)-Pinoresinol (5) An amorphous powder, $[\alpha]_{\mathrm{D}}^{25}+51^{c}\left(c=0.1, \mathrm{CHCl}_{3}\right) . \mathrm{UV} i_{\max }^{\mathrm{MeOH}} \mathrm{nm}: 232,281$. IR ${ }^{1}{ }_{\max }^{\mathrm{KBr}} \mathrm{cm}^{-1}: 3400(\mathrm{OH}), 1610,1510$ (aromatic ring). ${ }^{1} \mathrm{H}-\mathrm{NMR}$ (in $\left.\mathrm{CDCl}_{3}\right) \delta: 3.0-3.2\left(2 \mathrm{H}, \mathrm{m}, \mathrm{C}_{1.5}-\mathrm{H}\right), 3.87(6 \mathrm{H}, \mathrm{s}$, $\left.2 \times \mathrm{OCH}_{3}\right), 3.64-4.36\left(4 \mathrm{H}, \mathrm{m}, \mathrm{C}_{4.8}-\mathrm{H}\right), 4,73\left(2 \mathrm{H}, \mathrm{d}, J=5 \mathrm{~Hz}, \mathrm{C}_{2.6}-\mathrm{H}\right), 6.80-6.92\left(6 \mathrm{H}, \mathrm{m}\right.$, arom. H). ${ }^{13} \mathrm{C}-\mathrm{NMR}$ (in DMSO- $\left.d_{6}\right) \delta: 53.7(1,5), 55.8\left(\mathrm{OCH}_{3}\right), 71.0(4,8), 85.2(2,6), 110.6\left(2^{\prime}, 2^{\prime \prime}\right), 115.2\left(5^{\prime}, 5^{\prime \prime}\right), 118.6\left(6^{\prime}, 6^{\prime \prime}\right), 132.4\left(1^{\prime}\right.$, $\left.1^{\prime \prime}\right), 146.0\left(4^{\prime}, 4^{\prime \prime}\right), 147.6\left(3^{\prime}, 3^{\prime \prime}\right)$.

(+)-Medioresinol (6) An amorphous powder, $[\alpha]_{\mathrm{D}}^{25}+44.7^{\circ}(c=0.1, \mathrm{MeOH})$. UV $i_{\max }^{\mathrm{MeOH}} \mathrm{nm:} 231.5,280 . \mathrm{IR}$ $v_{\max }^{\mathrm{KBr}} \mathrm{cm}^{-1}: 3464(\mathrm{OH}), 1614,1518$ (aromatic ring). ${ }^{1} \mathrm{H}-\mathrm{NMR}$ (in DMSO-d $\left.d_{6}\right) \delta: 2.92-3.16\left(2 \mathrm{H}, \mathrm{m}, \mathrm{C}_{1.5}-\mathrm{H}\right), 3.76(9 \mathrm{H}$, $\left.\mathrm{s}, 3 \times \mathrm{OCH}_{3}\right), 3.80-4.40\left(4 \mathrm{H}, \mathrm{m}, \mathrm{C}_{4.8}-\mathrm{H}\right), 4.52-4.68\left(2 \mathrm{H}, \mathrm{m}, \mathrm{C}_{2.6}-\mathrm{H}\right), 6.52-6.92(5 \mathrm{H}, \mathrm{m}$, arom. H).

(+)-Syringaresinol (7)—Colorless needles (from EtOH), mp $183.5^{\circ} \mathrm{C} .[\alpha]_{\mathrm{D}}^{22}+44^{\circ}(c=0.1, \mathrm{MeOH})$. UV $i_{\max }^{\mathrm{MeOH}} \mathrm{nm}: 237,273 . \mathrm{IR} \mathrm{v}_{\max }^{\mathrm{KBr}} \mathrm{cm}^{-1}: 3428(\mathrm{OH}), 1614,1520$ (aromatic ring). ${ }^{1} \mathrm{H}-\mathrm{NMR}$ (in $\left.\mathrm{CDCl}_{3}\right) \delta: 2.92-3.18(2 \mathrm{H}$, $\left.\mathrm{m}, \mathrm{C}_{1.5}-\mathrm{H}\right), 3.64-4.27\left(4 \mathrm{H}, \mathrm{m}, \mathrm{C}_{4.8}-\mathrm{H}\right), 3.87\left(12 \mathrm{H}, \mathrm{s}, 4 \times \mathrm{OCH}_{3}\right), 4.61\left(2 \mathrm{H}, \mathrm{d}, J=4 \mathrm{~Hz}, \mathrm{C}_{2.6}-\mathrm{H}\right), 6.60(4 \mathrm{H}, \mathrm{s}$, arom. $\mathrm{H})$.

(+)-1-Hydroxypinoresinol (8)-An amorphous powder, $[\alpha]_{\mathrm{D}}^{25}+27^{\circ}(c=0.1, \mathrm{MeOH}) . \mathrm{UV} i_{\max }^{\mathrm{MeOH}} \mathrm{nm}: 232,281$. IR $v_{\max }^{\mathrm{KBr}} \mathrm{cm}^{-1}: 3440(\mathrm{OH}), 1616,1518$ (aromatic ring). ${ }^{1} \mathrm{H}-\mathrm{NMR}$ (in DMSO- $\left.d_{6}\right) \delta: 2.80-3.04\left(1 \mathrm{H}, \mathrm{m}, \mathrm{C}_{5}-\mathrm{H}\right), 3.46-$ $4.08\left(3 \mathrm{H}, \mathrm{m}, \mathrm{C}_{4 \mathrm{a}}-\mathrm{H}, \mathrm{C}_{8}-\mathrm{H}\right), 3.66\left(6 \mathrm{H}, \mathrm{s}, 2 \times \mathrm{OCH}_{3}\right), 4.20-4.48\left(1 \mathrm{H}, \mathrm{m}, \mathrm{C}_{4 \mathrm{e}}-\mathrm{H}\right), 4.52\left(1 \mathrm{H}, \mathrm{s}, \mathrm{C}_{2}-\mathrm{H}\right), 4.76(2 \mathrm{H}, \mathrm{d}$, $\left.J=5 \mathrm{~Hz}, \mathrm{C}_{6}-\mathrm{H}\right), 6.60-7.04(6 \mathrm{H}, \mathrm{m}$, arom. $\mathrm{H})$.

Caffeic Acid (10)-A white powder (from EtOH), mp 163.6 C. IR $v_{\max }^{\mathrm{KBr}} \mathrm{cm}^{-1}: 3440(\mathrm{OH}), 3000-2000,1646$ $(\mathrm{COOH}), 1620,1526$ (aromatic ring). ${ }^{1} \mathrm{H}-\mathrm{NMR}$ (in $\left.\mathrm{MeOH}-d_{4}\right) \delta: 6.20\left(1 \mathrm{H}, \mathrm{d}, J=16 \mathrm{~Hz}, \mathrm{C}_{\alpha}-\mathrm{H}\right), 6.60-7.10(3 \mathrm{H}, \mathrm{m}$, arom. $\mathrm{H}), 7.52\left(1 \mathrm{H}, \mathrm{d}, J=16 \mathrm{~Hz}, \mathrm{C}_{\beta}-\mathrm{H}\right) .{ }^{13} \mathrm{C}-\mathrm{NMR}$ (in MeOH- $\left.d_{4}\right) \delta: 115.2,115.7(2, \beta), 116.5(5), 122.7(6), 127.9(1)$, $146.6,146.9(3, \alpha), 149.2(4), 171.0(\%)$.

Methyl Chlorogenate (11) - A white powder (from EtOH). mp $100^{\circ} \mathrm{C} .[\alpha]_{\mathrm{D}}^{25}-26.4^{\circ}(c=0.1, \mathrm{MeOH})$. UV $i_{\max }^{\mathrm{MeOH}} \mathrm{nm}: 220,242,298,330 . \mathrm{IR} v_{\max }^{\mathrm{KBr}} \mathrm{cm}^{-1}: 3416(\mathrm{OH}), 1734,1694$ (COO), 1604, 1520 (aromatic ring). ${ }^{1} \mathrm{H}-\mathrm{NMR}$ (in $\left.\mathrm{MeOH}-d_{4}\right) \delta: 3.60\left(3 \mathrm{H}, \mathrm{s}, \mathrm{COOCH}_{3}\right), 5.20(1 \mathrm{H}, \mathrm{d}, J=6.4 \mathrm{~Hz}$, anomeric $\mathrm{H}), 6.10\left(1 \mathrm{H}, \mathrm{d}, J=16 \mathrm{~Hz}, \mathrm{C}_{\alpha}-\mathrm{H}\right), 6.60-7.00$ $\left(3 \mathrm{H}, \mathrm{m}\right.$, arom. H), $7.42\left(1 \mathrm{H}, \mathrm{d}, J=16 \mathrm{~Hz}, \mathrm{C}_{\beta}-\mathrm{H}\right) .{ }^{13} \mathrm{C}-\mathrm{NMR}$ (in $\left.\mathrm{MeOH}-d_{4}\right) \delta: 38.2\left(4^{\prime}, 6^{\prime}\right), 53.0\left(8^{\prime}\right), 70.7\left(3^{\prime}\right), 72.0$, $73.0\left(1^{\prime}, 2^{\prime}\right), 76.0\left(5^{\prime}\right), 115.2(2, \beta), 116.5(5), 122.9(6), 127.7(1), 146.6,147.0(3, \alpha), 149.4(4), 168.3(\gamma), 175.3\left(7^{\prime}\right)$. Acetylation of 11 gave a pentaacetate. A white powder, mp $167.9{ }^{\circ}{ }^{1}{ }^{1} \mathrm{H}-\mathrm{NMR}$ (in $\left.\mathrm{CDCl}_{3}\right) \delta: 2.01,2.10,2.16(9 \mathrm{H}$, each s, $3 \times$ alcoholic $\left.\mathrm{OCOCH}_{3}\right), 2.32\left(6 \mathrm{H}, \mathrm{s}, 2 \times\right.$ phenolic $\left.\mathrm{OCOCH}_{3}\right), 3.74\left(3 \mathrm{H}, \mathrm{s}, \mathrm{COOCH}_{3}\right), 6.32(1 \mathrm{H}, \mathrm{d}, J=16 \mathrm{~Hz}$, $\left.\mathrm{C}_{\alpha}-\mathrm{H}\right), 7.12-7.48(3 \mathrm{H}, \mathrm{m}$, arom. $\mathrm{H}), 7.60\left(1 \mathrm{H}, \mathrm{d}, J=16 \mathrm{~Hz}, \mathrm{C}_{\beta}-\mathrm{H}\right)$.

Genipin (12)—An amorphous powder, $[\alpha]_{\mathrm{D}}^{24}+95.4^{\circ}(c=1.0, \mathrm{MeOH})$. UV $i_{\max }^{\mathrm{MeOH}} \mathrm{nm}: 239 . \mathrm{IR} v_{\max }^{\mathrm{KBr}} \mathrm{cm}^{-1}: 3420$ $(\mathrm{OH}), 1692(\mathrm{C}=\mathrm{O})$. High-resolution MS $m / z: 226.0849\left(\mathrm{M}^{+}, \mathrm{C}_{11} \mathrm{H}_{14} \mathrm{O}_{5}\right), 208.0747\left(\mathrm{M}^{+}-\mathrm{H}_{2} \mathrm{O}, \mathrm{C}_{11} \mathrm{H}_{12} \mathrm{O}_{4}\right) .{ }^{1} \mathrm{H}-\mathrm{NMR}$ (in $\left.\mathrm{MeOH}-d_{4}\right) \delta ; 3.70\left(3 \mathrm{H}, \mathrm{s}, \mathrm{COOCH}_{3}\right), 4.22\left(2 \mathrm{H}, \mathrm{s}, \mathrm{C}_{10}-\mathrm{H}\right), 5.64-5.92\left(1 \mathrm{H}, \mathrm{m}, \mathrm{C}_{7}-\mathrm{H}\right), 7.48(1 \mathrm{H}, \mathrm{s}, \mathrm{C}-\mathrm{H})$.

Acknowledgements We wish to thank Mr. A. Sakushima of this university, and Asst. Prof. Dr. K. Takabe, Faculty of Engineering, Shizuoka University, for measurement of MS. Thanks are also due to Prof. Dr. T. Sasaya, Faculty of Agriculture, Hokkaido University, to Asst. Prof. Dr. F. Nakatsubo, Faculty of Agriculture, Kyoto University, and to Prof. Dr. T. Higuchi, Wood Research Institute, Kyoto University, for supplying charts of the spectra.

\section{References and Notes}

1) Part I: T. Deyama, Chem. Pharm. Bull., 31, 2993 (1983).

2) Part II: T. Deyama, T. Ikawa, and S. Nishibe, Chem. Pharm. Bull., 33, 3651 (1985).

3) Part III: T. Deyama, T. Ikawa, S. Kitagawa, and S. Nishibe, Chem. Pharm. Bull., 34, 523 (1986).

4) Part IV: T. Deyama, T. Ikawa, S. Kitagawa, and S. Nishibe, Chem. Pharm. Bull., 34, 4933 (1986).

5) a) T. Takehara and T. Sasaya, Mokuzai Gakkaishi, 25, 437, 660 (1979); b) H. D. Lüdemann and H. Ninz, Makromol. Chem., 175, 2393 (1974); c) P. K. Agrawal, R. P. Rastogi, and B. G. Osterdahl, Org. Magn. Reson., 21, 119 (1983).

6) H. Tsukamoto, S. Hisada, and S. Nishibe, Chem. Pharm. Bull., 32, 4653 (1984).

7) S. Omori and A. Sakakibara, Mokuzai Gakkaishi, 20, 388 (1974).

8) a) T. Sasaya, T. Takehara, and T. Kobayashi, Mokuzai Gakkaishi, 26, 759 (1980); b) T. Katayama, F. Nakatsubo, and T. Higuchi, ibid., 27, 223 (1981).

9) a) An authentic sample was purchased from Wako Pure Chemical Co.; b) M. Fukuoka, Chem. Pharm. Bull., 30 , 3219 (1982).

10) T. Okuda, T. Hatano, I. Agata, S. Nishibe, and K. Kimura, Abstracts of Papers, 102nd Annual Meeting of the Pharmaceutical Society of Japan, Osaka, April 1982, p. 553.

11) T. W. Bentley, R. A. W. Johnstone, and J. Grimshaw, J. Chem. Soc., 1967, 2234.

12) T. Kikuchi, S. Matsuda, S. Kadota, and T. Tai, Chem. Pharm. Bull., 33, 1444 (1984).

13) M. Haruna, T. Koube, K. Ito, and H. Murata, Chem. Pharm. Bull., 30, 1525 (1982).

14) a) Y. Sano and A. Sakakibara, Mokuzai Gakkaishi, 16, 81 (1970); b) A. Yamaguchi, Bull. Gov. For. Exp. Sta., 281, 1 (1976). 